\title{
Pacemaker electrode implantation in patients with persistent left superior vena cava
}

\author{
Franciszek Zerbe, Jacek Bornakowski, Wojciech Sarnowski
}

\begin{abstract}
Four out of 661 consecutive patients with permanent endocardial VVI pacing had the pacing lead introduced through a persistent left superior vena cava. It was difficult to introduce the lead from the right atrium into the right ventricle because the tip of the lead tended to be deflected away from the tricuspid orifice. This difficulty was overcome by shaping the lead into a pigtail with $3-4 \mathrm{~cm}$ wide loop. This avoided the risk of entering a branch of the coronary sinus in order to reach the right atrium.

This technique made it easier to position the lead in the right ventricular apex. There were no complications in these four patients during a mean follow up of three years.
\end{abstract}

Placement of pacemaker electrodes can be complicated by congenital anomalies of the systemic superior veins, including a persistent left superior vena cava with normal right superior vena cava (a double system), a single left superior vena cava, and absence of drainage by a superior vena cava. ${ }^{1}$

A persistent left superior vena cava arises from the confluence of the left jugular and

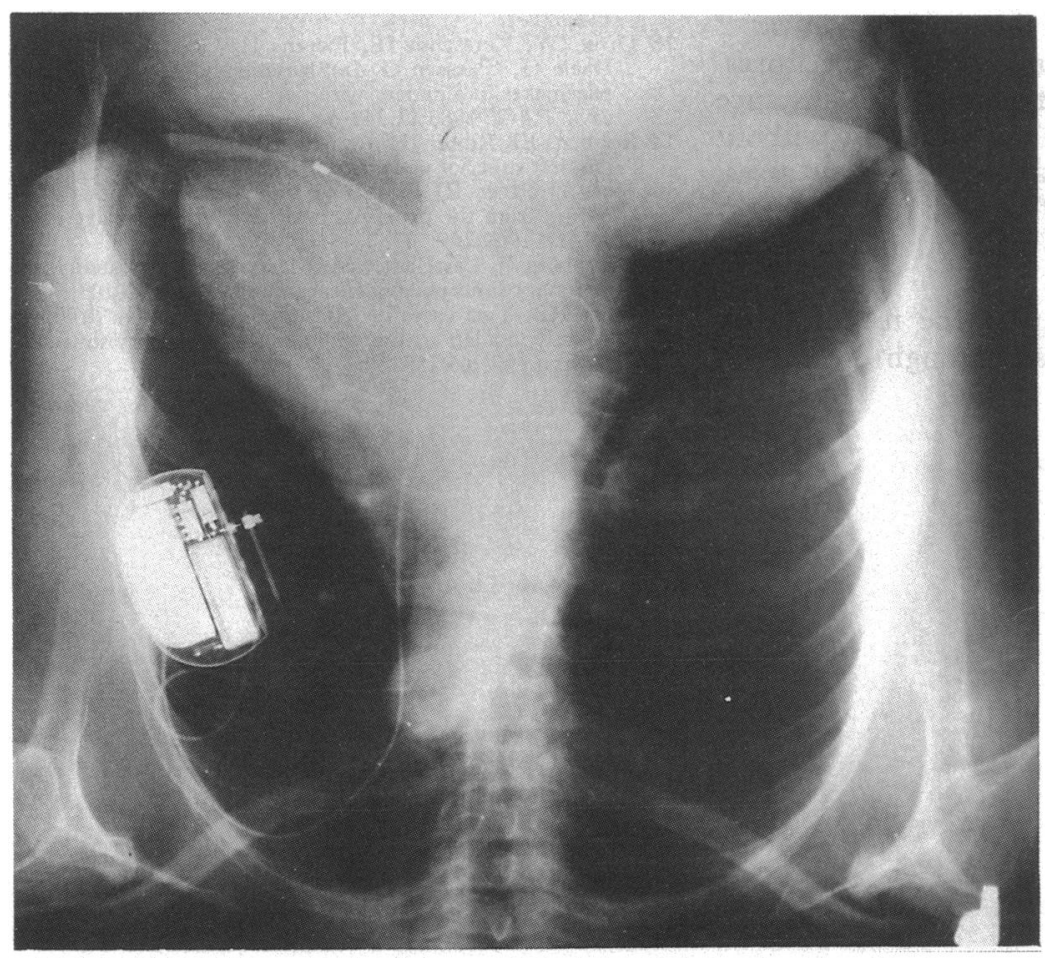

Course of the pacemaker lead introduced through persistent left superior vena cava in patient 4. subclavian veins and passes down to enter the right atrium through the coronary sinus. Such an arrangement is seldom recognised on the plain chest $\mathrm{x}$ ray but because of the unusual course of a lead (figure) it becomes obvious on the screen when a pacemaker lead is being introduced. Once the right atrium is reached through the coronary sinus further placement of a lead in the right ventricle can be difficult because the tip of the lead is deflected away from the tricuspid orifice.

\section{Patients and methods}

Among 661 patients who had permanent unipolar pacemaker (VVI) leads inserted from a branch of the left subclavian vein between January 1982 and July 1990 there were four patients in whom the pacemaker lead was introduced through the persistent left superior vena cava (table). In all four the unusual left-sided downward course taken by the electrode lead during the procedure alerted us to the presence of a persistent left superior vena cava. We found that if we reshaped the end of the stylet into the pigtail configuration we were able to place the lead in the right ventricle without difficulty.

In three of the four patients we also had to change to a curved stylet before the tip of the lead could be positioned in the right ventricular apex. During a mean follow up of $35 \cdot 2$ months there were no complications.

\section{Discussion}

During fetal life the superior vena cava develops from the right anterior and common cardinal veins. The left anterior cardinal vein becomes the proximal parts of the left internal jugular and subclavian veins draining through the anastomotic channel (left brachiocephalic vein) into the superior vena cava. The left common cardinal vein and distal left sinus horn become the left superior vena cava (draining to the coronary sinus) which normally regresses, its site being marked by the oblique ligament and vein of Marshall. If this process is interrupted a double caval system develops. Other much rarer anomalies that can affect pacemaker lead insertion include single left persistent vena cava or even the complete lack of superior caval drainage. ${ }^{1}$ Such anomalies are seldom found without other congenital defects. For instance, the estimated incidence of a double superior caval system in the general population is $0.1 \%$ $0.5 \% .^{23}$

Anomalies of the superior caval system can 
Details of patients with pacemaker leads implanted through a persistent left superior vena cava

\begin{tabular}{lllll}
\hline Patient & $\begin{array}{l}\text { Age at } \\
\text { implantation, sex }\end{array}$ & Indication for pacing & $\begin{array}{l}\text { Follow up } \\
(\text { mnth })^{\star}\end{array}$ & Outcome \\
\hline 1 & $63, \mathrm{~F}$ & $2^{\circ}$ AV block & 22 & Lost to follow up \\
2 & $73, \mathrm{M}$ & $3^{\circ}$ AV block & 49 & Alive \\
3 & $76, \mathrm{~F}$ & $2^{\circ}$ AV block & 16 & Died, cause unknown \\
4 & $55, \mathrm{~F}$ & SSS (sinus bradycardia) & 54 & Alive \\
\hline
\end{tabular}

^Mean 35.2 months.

AV, atrioventricular; SSS, sick sinus syndrome.

be diagnosed by conventional angiography, by cross sectional echocardiography combined with peripheral venous injection of contrast medium, ${ }^{4}$ by intravenous digital subtraction angiography, ${ }^{5}$ by radionuclide angiography, ${ }^{6}$ and by magnetic resonance imaging. ${ }^{7}$

Attempts to define the anatomy of the superior caval system before pacemaker implantation are not routine because anomalies are rare and because the most commonly encountered anomaly, a persistent left superior vena cava, does not preclude successful lead placement.

Many isolated cases have been reported and Trigano reviewed 39 cases. $^{3}$ Experience indicates that it is possible to introduce a pacemaker lead into the right ventricle for effective pacing in one chamber pacing ${ }^{8-10}$ and dual chamber pacing ${ }^{11-13}$ and into the right atrium for atrial pacing. ${ }^{14}$

When the pacemaker lead enters the right atrium through the persistent left superior vena cava and the coronary sinus it often forms a loop in the right atrium and then enters the right ventricle. Rarely a lead may reach the right ventricle without forming a loop or enter a branch of the coronary sinus; these two courses are sometimes difficult to distinguish in the anterior-posterior view. ${ }^{3}$ Fortunately, ventricular pacing (usually from a branch of the coronary sinus) and atrial pacing (from the distal coronary sinus) $)^{1516}$ seem to be safe long term procedures. Appropriate curving of a stylet, for instance into a pigtail loop 3-4 cm wide but without complete "closing" of a loop, as in our cases, facilitated insertion of a lead to the right ventricle by directing the lead into the tricuspid orifice.

It is important to know the technique of implanting a pacing lead through the persis- tent left superior vena cava because in patients with a single left superior vena cava the only alternative is the transthoracic approach with all its inherent risks. The transthoracic approach seems to be mandatory only in those very rare cases of a single persistent left superior vena cava draining to the left atrium. ${ }^{17}$ Such a case has not been so far described in connection with pacemaker lead implantation. There is no need to use active fixation leads with screw-in or pinch-in tips.

1 Amjad Hussein S, Chakravarty S, Chaikhouni A, Smith JR Congenital absence of superior vena cava: unusual anomaly of superior systemic vein complicating pacemaker placement. $P A C E$ 1981;4:328-32.

2 Steinberg I, Dubilier W, Lukas D. Persistence of lef superior vena cava. Dis Chest 1953;24:479-82.

3 Trigano JA. Permanent pacing through a persistent left superior vena cava in 39 collected cases. Clin Prog Electrophysiol Pacing 1986;4:45-52.

4 Foale $R$, Bourdillon PD, Somerville J, Rickards A Anomalous systemic venous return: recognition by twodimensional echocardiography. Eur Heart J 1983;4: 186-95.

5 Yiannikas J, Moodie DS, Gill CC, Sterba R, McIntyre R, Buonocore E. Intravenous digital subtraction angiography in the assessment of patients with left to right sraphy in the assessment of patients with left to right Cardiol 1984;3:1507-14.

6 Otto AC, Answegen A, Herbst CP, et al. Persistent left superior vena cava detected with radionuclide angiocardiography. Clin Nucl Med 1985;10:27-9.

7 Fisher MR, Hricak H, Higgins CB. Magnetic resonance imaging of developmental venous anomalies. Am J Roentgenol 1985;145:705-9.

8 Rose ME, Gross L, Protos A. Transvenous pacemake implantation by way of an anomalous left superior ven cava. $J$ Thorac Cardiovasc Surg 1971;62:965-6.

9 Harris A, Gialafos J, Jefferson K. Transvenous pacing in presence of anomalous venous return to heart. Br Heart 1972;34:1189-91.

10 Gillmer DJ, Vyhilingum S, Mitha AS. Problems encountered during insertion of permanent endocardial pacing tered during insertion of perman

11 Westerman GR, Baker J, Dungan WT, Van Devanter SH Permanent pacing through a persistent superior vena cava: an aproach and report of dual-chambered lead an approach and report of dual-chamber

12 Zardo F, Nicolosi GL, Burelli C, Zanuttini D. Dualchamber transvenous pacemaker implantation via anomalous left superior vena cava. Am Heart J 1986, $112 ; 621-2$.

13 Dirix LY, Kersschot IE, Fierens H, Goethals MA, Van Daele G, Claessen G. Implantation of a dual-chamber pacemaker in a patient with persistent left superior ven cava. $P A C E$ 1988;11:343-5.

14 Robbens EJ, Ruiter JH. Atrial pacing via unilateral persistent left superior vena cava. $P A C E$ 1986;9:594-6.

15 Moss AJ, Rivers RJ, Cooper M. Long-term pervenous atria pacing from the proximal portion of the coronary vein. pacing from the proximal

16 Greenberg P, Castellanet M, Messenger J, Ellestad MH. Coronary sinus pacing. Circulation 1978;57:98-103.

17 Wiles HB. Two cases of left superior vena cava draining directly to a left atrium with a normal coronary sinus. $B r$ Heart J 1991;65:158-60. 